\title{
A New Rush Schedule for Cotrimoxazole Desensitization: A Report of 2 Cases
}

Rial Prado $\mathrm{MJ}^{1}$, Rico Díaz $\mathrm{MA}^{2}$, Cosgaya Ceballos $\mathrm{A}^{1}$, Cuesta Herranz $\mathrm{J}^{1}$

${ }^{1}$ Hospital Universitario Fundación Jiménez Díaz, Madrid, Spain ${ }^{2}$ Complexo Hospitalario Universitario A Coruña, A Coruña, Spain

J Investig Allergol Clin Immunol 2018; Vol. 28(4): 267-269 doi: $10.18176 /$ jiaci.0256

Key words: Desensitization. Cotrimoxazole. Drug allergy. Sulfonamide hypersensitivity.

Palabras clave: Desensibilización. Cotrimoxazol. Alergia a medicamentos. Alergia a sulfamidas.

Trimethoprim-sulfamethoxazole (cotrimoxazole) is an effective drug for the treatment of infectious diseases caused by gram-positive bacteria, gram-negative bacteria, and protozoa that reduces the risk of opportunistic infection by Pneumocystis jiroveci [1]. Sulfonamides are the most common culprits of adverse reactions to cotrimoxazole [2]. The prevalence of adverse reactions to cotrimoxazole ranges from $20 \%$ to $100 \%$ in certain populations, such as HIV-infected patients, while in healthy individuals the prevalence is normally between $5 \%$ and $8 \%$ [3]. The 2 possible therapeutic approaches following confirmed diagnosis of adverse reactions to cotrimoxazole are withdrawal of the drug and desensitization. We report 2 cases in which a new rush desensitization schedule for cotrimoxazole was used. A maintenance dose was achieved in 135 minutes, thus enabling a regimen that could be administered on alternate days.

The first case involved a 67-year-old white woman with stage IIIA follicular lymphoma. Approximately 4 months before presenting at our department, she was prescribed cotrimoxazole on alternate days as prophylactic treatment for Pjiroveci infection. A few days after beginning treatment, she presented generalized itchy rash, mainly on her legs, which resolved with antihistamines and withdrawal of cotrimoxazole. For this reason, the patient was referred to our allergy department, where skin prick tests were performed with trimethoprim at 32 $\mathrm{mg} / \mathrm{mL}$ and sulfamethoxazole at $200 \mathrm{mg} / \mathrm{mL}$ (Almofarma SL) and intradermal tests at $0.001 \mathrm{mg} / \mathrm{mL}$ of trimethoprim and $20 \mathrm{mg} / \mathrm{mL}$ of sulfamethoxazole, as previously reported [3]. The results were negative. An oral challenge test elicited a generalized exanthematous rash 30 minutes after a dose of 200/40 mg of trimethoprimsulfamethoxazole. Desensitization to cotrimoxazole was indicated, as this was the only oral antimicrobial available for prophylaxis of $P$ jiroveci.

Desensitization was performed using a new rush intravenous desensitization protocol based on the protocol of Gluckstein and Ruskin [4] (Table), with good tolerance. The procedure was performed at the hospital, with a physician and nurse in attendance and emergency medication readily available. Written informed consent (both for the challenge test and desensitization procedure) was given by 
Table. Intravenous Desensitization Protocol

\begin{tabular}{lccccccc} 
Steps & Rate & $\begin{array}{c}\text { Time, } \\
\text { min }\end{array}$ & $\begin{array}{c}\text { Cumulative } \\
\text { Time, min }\end{array}$ & $\begin{array}{c}\text { Volume } \\
\text { Administered, mL }\end{array}$ & $\begin{array}{c}\text { Dose Administered, } \\
\mathrm{mg}^{\mathrm{a}}\end{array}$ & $\begin{array}{c}\text { Cumulative Dose, } \\
\mathrm{mg}\end{array}$ \\
\hline 1 & Bolus & 15 & 15 & $1 \mathrm{~mL}$ & $0.02 / 0.004$ & $0.02 / 0.004$ & $0.02 / 0.004 \mathrm{mg} / \mathrm{mL}$ \\
2 & Bolus & 15 & 30 & $1 \mathrm{~mL}$ & $0.2 / 0.04$ & $0.22 / 0.044$ & $0.2 / 0.04 \mathrm{mg} / \mathrm{mL}$ \\
3 & Bolus & 15 & 45 & $1 \mathrm{~mL}$ & $2 / 0.4$ & $2.22 / 0.444$ & $2 / 0.4 \mathrm{mg} / \mathrm{mL}$ \\
4 & $40 \mathrm{~mL} / \mathrm{h}$ & 15 & 60 & $10 \mathrm{~mL}$ & $20 / 4$ & $22.22 / 4.444$ & $2 / 0.4 \mathrm{mg} / \mathrm{mL}$ \\
5 & $200 \mathrm{~mL} / \mathrm{h}$ & 30 & 90 & $100 \mathrm{~mL}$ & $200 / 40$ & $22.22 / 44.444$ & $2 / 0.4 \mathrm{mg} / \mathrm{mL}$ \\
6 & $133 \mathrm{~mL} / \mathrm{h}$ & 45 & 135 & $100 \mathrm{~mL}$ & $800 / 160$ & $1022.22 / 204.444$ & $8 / 1.6 \mathrm{mg} / \mathrm{mL}$ \\
\hline
\end{tabular}

${ }^{a}$ After the last dose is administered, the patient is kept under observation for 2 hours

the patient following an explanation of the risks involved. As the recommended regimen for long-term prophylaxis of pneumocystis is administration of the drug every 48 hours, a challenge with cotrimoxazole was performed at 48 hours after desensitization, with no adverse reaction. Cotrimoxazole at was prescribed at $800 / 160 \mathrm{mg}$ every 48 hours uninterruptedly for the next 6 months to maintain the tolerance acquired.

The second case involved a 26-year-old white woman who had undergone lung transplantation owing to cystic fibrosis. She had experienced generalized urticaria within 72 hours of beginning treatment with trimethoprim-sulfamethoxazole, which was prescribed as in the previous case for prophylaxis of $P$ jiroveci. The results of skin prick testing were negative. We decided to perform an oral challenge test, which induced hives after 40 minutes of administration at a dose of 100/20 mg. Once a diagnosis of adverse reaction to cotrimoxazole was established, we decided to perform desensitization with the protocol described in the previous case (Table). The patient tolerated the protocol well. After 48 hours of desensitization, provocation in the allergy clinic caused no adverse reactions. Once it was established that the patient tolerated the doses well, we indicated that she should continue home administration of cotrimoxazole at a dose of $800 / 160 \mathrm{mg}$ every 48 hours. She experienced no adverse reactions over the full 6-month period indicated for posttransplant pulmonary prophylaxis.

Desensitization protocols have been developed to safely reintroduce critical drugs in patients with prior reactions to these drugs. The mechanisms of desensitization are still unknown [5]. Several protocols for desensitization to cotrimoxazole have been described. These can last from a single day to several days, the shortest being from 90 minutes to 6 hours [6]. Most of the protocols described involve oral administration and are normally used in adults, although protocols have also been described in children $[7,8]$. Pyle et al [6] reported the safety and efficacy of outpatient administration of cotrimoxazole in immunocompetent persons with a history of adverse reaction to sulfonamides. Our protocol is one of the fastest published to date, and is original in that it is intravenous, which has the advantage of more accurate dosing. Additionally, protocols such as ours may be suspended in the case of adverse effects, although very rapid administration can cause undesirable effects. We administer the dose corresponding to each of the first 3 steps in a milliliter bolus. The last 2 doses are given slowly in a volume of $100 \mathrm{~mL}$.
After the last dose is administered, the patient is kept under observation for 2 hours.

To the best of our knowledge, there are 3 noteworthy factors reported in these cases. First, the new rapid intravenous protocol used allows the maintenance dose to be achieved in only 135 minutes. The time between each dose was 15 minutes and was derived by modifying the oral regimen described by Gluckstein and Ruskin [4] in 1995. The doses were administered in such a way as to bring the pattern closer to that used in the desensitization of cytostatic agents. The absence of adverse effects in both cases seems to indicate that the protocol used was safe. Second, the adverse reaction to cotrimoxazole was confirmed by means of a positive challenge test. Finally, as the pneumocystis prophylaxis regimen (maintenance dose) was established for alternate days (every 48 hours), a controlled rechallenge was performed, with good tolerance in both cases. Nonetheless, we report only 2 cases; further experience is needed before our protocol can be used for routine desensitization.

In summary, we report a new rush schedule for intravenous desensitization that is safe and effective and allows for maintenance doses to be administered on alternate days. The desensitization protocol we describe can benefit patients who have adverse reactions to cotrimoxazole and need both a rush protocol and maintenance doses every 48 hours.

\section{Acknowledgments}

We thank Oliver Shaw for his assistance in editing the English version.

\section{Funding}

The authors declare that no funding was received for the present study.

\section{Conflicts of Interest}

The authors declare that they have no conflicts of interest.

\section{References}

1. Maertens J, Cesaro S, Maschmeyer G, Einsele H, Donnelly JP, Alanio A et al. ECIL guidelines for preventing Pneumocystis jirovecii pneumonia in patients with haematological 
malignancies and stem cell transplant recipients. J Antimicrob Chemother. 2016 Sep;71(9):2397-04.

2. Moreno Escobosa MC, Cruz Granados S, Moya Quesada MC, Amat Lopez J. Enanthema and fixed drug eruption caused by trimethoprim. J Invest Allergol Clin Immunol. 2009;19:23752.

3. Choquet-Kastylevsky G, Vial T, Descotes J. Allergic adverse reactions to sulfonamides. Curr Allergy Asthma Rep. 2002;2:16-25.

4. Gluckstein D, Ruskin J. Rapid oral desensitization to trimethoprim-sulfamethoxazole (TMP-SMZ): use in prophylaxis for Pneumocystis carinii pneumonia in patients with AIDS who were previously intolerant to TMP-SMZ. Clin Infect Dis. 1995Apr;20(4):849-53.

5. De las Vecillas L, Alenazy LA, Garcia-Neuer M, Castells M. Drug Hypersensitivity and Desensitizations: Mechanisms and New Approaches. Int J Mol Sci. 2017;18:1316.

6. Pyle RC, Butterfield JH, Volcheck GW, et al. Successful outpatient graded administration of trimethoprimsulfamethoxazole in patients without HIV and with a history of sulfonamide adverse drug reaction. J Allergy Clin Immunol Pract. 2014;2:52-8.

7. Gómez-Traseira C, Boyano-MartínezT, Escosa-García L, Pedrosa M, Martín-Muñoz F, Quirce S. Trimethoprim-sulfamethoxazole (cotrimoxazole) desensitization in an HIV-infected 5-yr-old girl. Pediatr Allergy Immunol. 2015 May;26(3):287-9.

8. D'Amelio CM, Del Pozo JL, Vega O, Madamba R, Gastaminza $G$. Successful desensitization in a child with delayed cotrimoxazole hypersensitivity: A case report. Pediatr Allergy Immunol. 2016 May;27(3):320-1.

1 Manuscript received December 27, 2017; accepted for publication March 26, 2018.

\section{Manuel Jorge Rial Prado}

Allergy Department

Hospital Universitario Fundación Jiménez Díaz Avenida de los Reyes Católicos, 2

28040, Madrid, Spain

E-mail: manuel.rial@quironsalud.es 